\section{Einfluss von Malokklusion, Saughabits und Karies auf die Kaufunktion von Vorschulkindern}

Souto-Souza D et al. The influence of malocclusion, sucking habits and dental caries in the masticatory function of preschool children. Braz Oral Res 2020. doi:10.1590/1807-3107bor2020.vol34.0059

Die Nahrungszerkleinerung während des Kauens erleichtert die Aufnahme von Nährstoffen, was für Wachstum und Entwicklung eines Kindes von grundlegender Bedeutung ist. Studien haben gezeigt, dass ein höherer BodyMass-Index, Karies, häufige Aufnahme von pastösen Nahrungsmitteln, wenige Kaueinheiten, eine geringere Bewegung des Unterkiefers und eine Malokklusion verbunden sind mit einer schlechten Kaufunktion.

Vergleicht man diese Faktoren, findet man in der Literatur kontroverse Ergebnisse zur Rolle der Malokklusion. Die Prävalenz von Malokklusion kann bei Vorschulkindern bis zu 87,0\% erreichen; die hohe Prävalenz könnte in Zusammenhang stehen mit nicht nahrungsbezogenen Sauggewohnheiten. Längerer Gebrauch einer Saugflasche wird mit übermäßiger Milchaufnahme in Verbindung gebracht. Dieser höhere Milchkonsum kann zu einer geringeren Vorliebe des Kindes für festere Nahrungsmittel wie Obst und Fleisch beitragen. Ziel einer aktuellen Studie von Débora Souto-
Souza von der UFVJM Universität in Diamantina, Brasilien, und Kollegen war es, den Zusammenhang zwischen Malokklusion, ernährungsbezogenen und nicht nahrungsbezogenen Saughabits und der Kaufunktion bei Vorschulkindern zu untersuchen.

Dazu führte sie eine Querschnittsstudie mit 384 Kindern im Alter von 3 bis 5 Jahren durch. Das Vorliegen einer Malokklusion wurde nach den Foster- und Hamilton-Kriterien ermittelt. Die Anzahl der Kaueinheiten und der von Karies befallenen Backenzähne wurde ebenfalls erfasst. Die Eltern beantworteten einen Fragebogen, der Fragen zu den ernährungsbezogenen und nicht nahrungsbezogenen Sauggewohnheiten des Kindes enthielt. Die Kaufunktion wurde mit Optocal-Testmaterial evaluiert und basierte auf der mittleren Teilchengröße in der Kauleistung, auf der Schluckschwelle und auf der Anzahl der Kauzyklen während der Schluckschwelle.

Von den 384 Kindern hatten 206 eine Malokklusion, 178 nicht. Ihr Durchschnittsalter betrug 4,2 Jahre. Was die Sauggewohnheiten betraf, so wurden 16,9\% mit der Flasche gefüttert, und 34,1\% verwendeten aktuell oder in der Vergangenheit einen Schnuller. Die Anzahl der Kaueinheiten reichte von 1 bis 4 (Mittelwert 3,92). Die Prävalenz von Karies im Seitenzahnbereich betrug $37 \%$.

Die Kaufunktion wurde mit einer mittleren Partikelgröße von 5,1 mm in der Kauleistung, einer Schluckschwelle von 4,2 mm und der mittleren Anzahl an durchgeführten Kauzyklen bis zur Schluckschwelle von 30,7 Zyklen angegeben.

Im finalen multiplen Regressionsmodell waren die mediane Partikelgröße in der Kauleistung und die Schluckschwelle assoziiert mit dem Alter, mit Flaschenfütterung und mit einer posterioren Zahnfehlstellung. Die Kauleistung wurde auch mit der Anzahl der kavitierten Seitenzähne in Verbindung gebracht.

Die Schluckschwelle war mit dem Alter, der Flaschenfütterung und der posterioren Malokklusion assoziiert. Die Anzahl der Kauzyklen während der Schluckschwelle war mit der Anzahl der kavitierten Seitenzähne assoziiert.

FAZIT

Die vorliegende Studie zeigte, dass jüngere Kinder mit einer Vorgeschichte von Flaschenernährung und nachfolgender Malokklusion größere Schwierigkeiten hatten, die Testnahrung entsprechend ihrer Kauleistung und Schluckschwelle in kleinere Partikel zu zerlegen. Darüber hinaus führten Kinder mit einer höheren Anzahl kavitierter Backenzähne eine größere Anzahl von Kauzyklen durch, bevor sie die Schluckschwelle erreichten, und hatten eine schlechtere Kauleistung.

Dr. Michaela Bitzer, Tübingen 